\title{
Research article \\ Seroprevalence of antibodies against varicella among medical students - a need for immunization record for medical school admission
}

\author{
Malay Jhancy ${ }^{1}$, Grisilda Vidya Bernhardt ${ }^{2}$, Maryam Risla ${ }^{3}$, Munazza Khanam ${ }^{4}$, Syeda Sana Sultana ${ }^{5}$ \\ ${ }^{1}$ Associate Professor, Department of Pediatrics, ${ }^{2}$ Associate Professor, Department of Biochemistry, RAK Medical and Health \\ Sciences University, ${ }^{3,4,5}$ Interns, SAQR Hospital, RAKMHSU, Ras Al Khaimah, UAE
}

(Received: January $2021 \quad$ Revised: February $2021 \quad$ Accepted: March 2021)

Corresponding author: Malay Jhancy. Email: jhancy@rakmhsu.ac.ae

\begin{abstract}
Introduction and Aim: This study was conducted at a medical University in UAE to assess the risk of acquiring Varicella - a vaccine-preventable disease, by estimating antibody titers among the clinically exposed students to provide a scientific implementation of immunization guideline during admission to medical school.

Materials and Methods: This cross-sectional study involved 88 students in clinical years attending a medical college in the UAE. The students' demographics, vaccination data, and history of natural infection with vaccine-preventable communicable infectious diseases were collected, with more significance on Varicella. Blood samples were collected to determine varicella IgG via ELISA.
\end{abstract}

Results: Out of 88 medical students, $20.45 \%$ of students had vaccination during childhood and $60.22 \%$ of students had a natural infection in the last five years. Three students $(3.4 \%)$, who had childhood vaccination acquired the infection which is statistically significant during their clinical study years. $70.4 \%$ of students who had childhood vaccination and natural infection are at risk of acquiring varicella infections due to the waning of antibodies. $13.64 \%$ were found to be negative for varicella antibody titers are at the highest risk.

Conclusion: Medical students are most susceptible to acquiring the infection. Medical Universities should take up a policy, that each student needs to take booster vaccinations as a mandatory requirement before admission into the first year, which is practiced as an admission protocol in Western countries like the United States of America but not in Asian countries. Special emphasis should be placed on catch-up vaccination of students because the likelihood of severe infection increases with age.

Keywords: Risk of infection; medical students in the clinical year of study; protective antibodies; booster vaccination; immunization status criteria for medical school admission.

\section{INTRODUCTION}

$\mathrm{V}$ aricella-zoster virus (VZV) is a human herpesvirus (HHV); known as HHV-3, is a member of the DNA-containing the Herpesviridae family. VZV causes varicella (chickenpox) as a primary infection and herpes zoster (HZ) after the reactivation of a latent VZV. It is transmitted by respiratory aerosols or direct skin contact from the affected individuals (1). VZV infection is very contagious and characterized by a generalized exanthematous rash and a period of morbidity for about two weeks. Varicella is a selflimited infection in immunocompetent children but causes severe infection in adults. For the normal nonimmunized individual, chickenpox-associated mortality is less than two per 100,000 . This risk increases manifold for adults as the general symptoms and skin lesions are more problematic and complications (e.g., pneumonia, encephalitis, and hepatitis) are more often fatal in adults and adolescents $(2,3)$.

The Immunoglobulins produced by our body in response to an antigen are responsible for fighting the disease and providing immunity. IgM is the largest immunoglobulin having pentamer units and ten antigen-binding sites that appear first whenever a foreign antigen enters the blood for the first time. IgG antibodies appear later and are responsible for humoral immunity. IgG is a monomer unit that is lighter and smaller, with two antigen-binding sites. Due to the smaller size of this antibody, it can travel through the bloodstream $(4,5)$.

Live attenuated vaccines fight viruses and bacteria by providing continual antigenic stimulation giving sufficient time for memory cell production. These vaccines contain an inactive part of the viruses or bacteria that mimic natural infection, without causing disease activates immune systems. Measles, mumps, and rubella vaccine (MMR) and varicella (chickenpox) vaccine are some of the live attenuated vaccines. Unless these vaccines are included in vaccination programs and insist on boosters, not everyone takes these vaccines. Children with weakened immune systems - for example, those who are undergoing chemotherapy-can not get live vaccines $(5,6)$.

Immunity against vaccine-preventable and other transmissible infections is of major importance, 
especially in medical students who are at increased risk of exposure to infectious body fluids. Thus, uniform medical school admission policies to screen the students for protective antibodies for common infectious diseases are desirable implementation of cost-effective programs, however, require surveillance that identifies immunization gaps and susceptibility to infectious diseases (7).

The vaccine against varicella was included in the UAE immunization program in the year 2012, although the vaccine has been available in the UAE before that. A live, two-dose attenuated varicella vaccine is recommended for all children. UAE is one of the first countries in the Gulf to have adopted the WHO Expanded Program on Immunization (EPI). Therefore, childhood vaccine-preventable diseases have drastically reduced. But, one can never be sure of complete immunity against some of these infections as the protection weans over time, which results in unsustainable protection in adults $(8,9)$.

The first dose should be administered at the age of 1215 months and the second dose at 5-6 years. Varicella vaccine is $85 \%$ effective in preventing any disease and $97 \%$ effective in preventing moderately severe and severe disease. Medical students are exposed to various infections during their training period and are susceptible to some vaccine-preventable viral infections if they aren't immune to it (9). Over the years, the antibody titers against varicella have been shown to recede (10). This makes it very important to check the protective antibody levels, especially in the health care workers.

Medical students were found to have low immunity against vaccine-preventable diseases as highlighted in a recent review of the literature on vaccination among healthcare students $(11,12)$. A study conducted on immunization of medical students between 2002 and 2005 using questionnaires and serological tests concluded that a significant portion of first-year medical students was not immune to important vaccine-preventable diseases (13).

Catch-up vaccination for medical students , susceptible older children is important to prevent the accumulation of susceptibility in these age groups, in which the natural disease is more severe due to high chances of disease complications. These students are also at risk of future exposure to the disease and could potentially be a source of infection transmission to peers and patients. Thus study exploring importance of medical School entry requirements of booster vaccination will contribute to more rapid implementation of the existing recommendations for vaccination (12).

\section{METHODOLOGY}

This was a cross-sectional study conducted at a medical University in the UAE within the course of 4 months (January 2019 - April 2019). Vaccination history was taken using a questionnaire and the seroprotective antibody titers against varicella were estimated by ELISA.

A total of 88 medical students from clinical years (years 3, 4, 5 of MBBS) participated in this study. None of them had any significant medical diseases and were not on any immunocompromised medication. Consent was obtained from every participant. Ethical clearance was obtained before commencement of the study (RAKMHSU Institutional ethics committee, reference number : REC-50-2018-UG-M and Ministry of Health and Prevention Research Ethics Committee/RAK Subcommittee reference number : MOHAP/REC/2019/03-2019-UG-M dated $24^{\text {th }}$ January 2019).

Vaccination history was taken by a questionnaire that included demographics, recent and past infection status, details of vaccination history, history of exposure to disease, and natural infection.

To determine the protective antibody titers against varicella, blood samples were collected with aseptic precautions. Blood was centrifuged to separate the serum and was serum was stored at $-20^{\circ} \mathrm{C}$ for the determination of varicella IgG. Protective antibody levels in the sera of the study candidates were quantitated by ELISA.

VIRCELL varicella ELISA kit was used to determine the IgG levels in the samples based on the reaction of antibodies in the sample that is to be tested with the antigen that is absorbed on the polystyrene surface, kit procedure of followed at every step. Final Optical density was measured using ELISA plate reader at $450 / 620 \mathrm{~nm}$.

Results were then interpreted by calculating the mean optical density (O.D.) for cut off of serum. The Antibody index $=$ (sample O.D./ cut off serum mean O.D.) $x 10$ is calculated. The antibody index less than 9 was considered as not having IgG-specific antibodies against varicella, between 9 to 11 is taken as equivocal hence the test was repeated, and more than 11 were taken as positive with sufficient IgG varicella antibodies.

\section{RESULTS}

Out of 88 participanting, medical students from the $3^{\text {rd }}, 4^{\text {th, }}$ and $5^{\text {th }}$ clinical years (Graph 1$), 56.81 \%$ were girls and $43.18 \%$ were boys (Graph 2). Students from 11 different nationalities participated in the study (Table 1). 


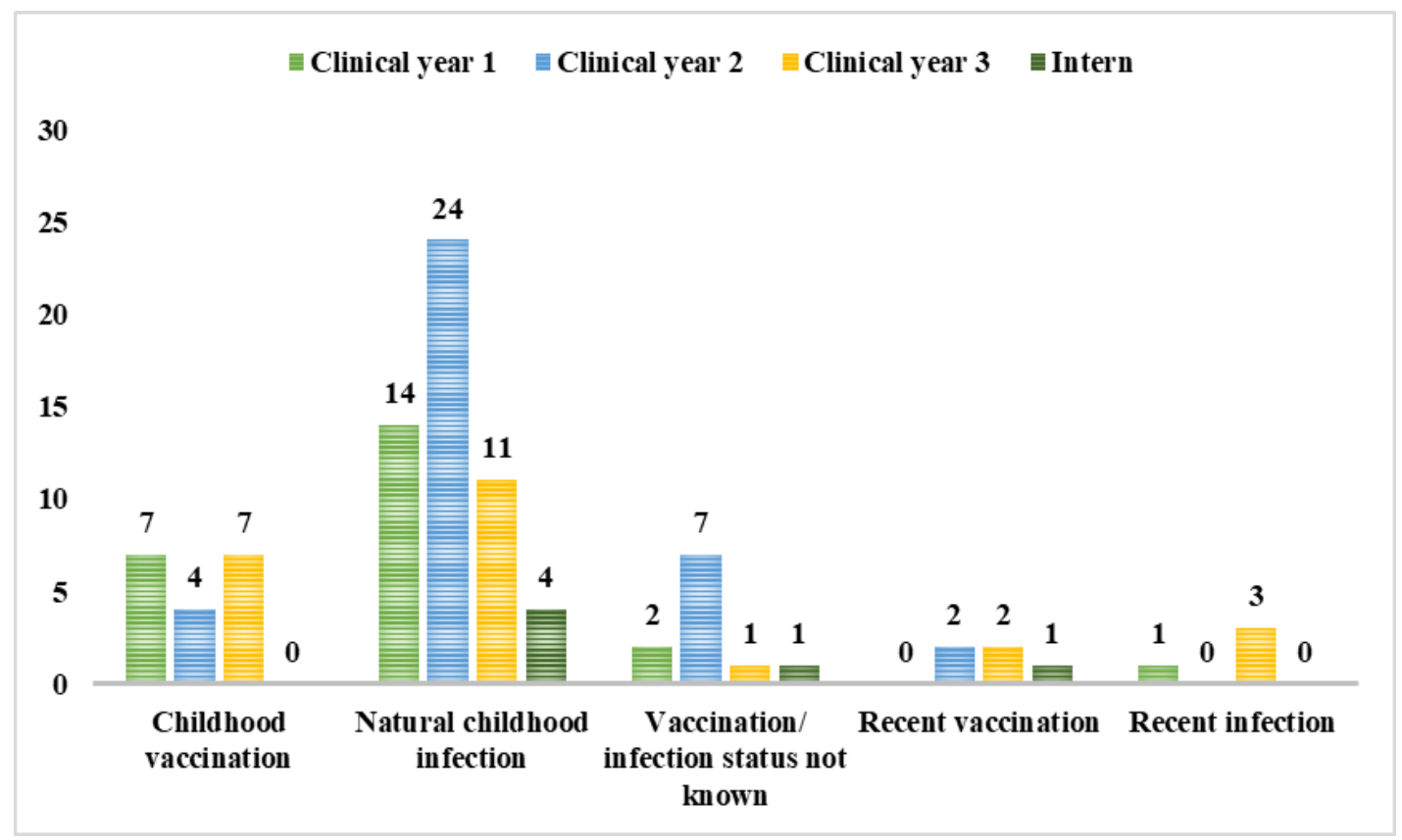

Graph 1: Comparison of clinical years with regards to vaccination and infection status of students.

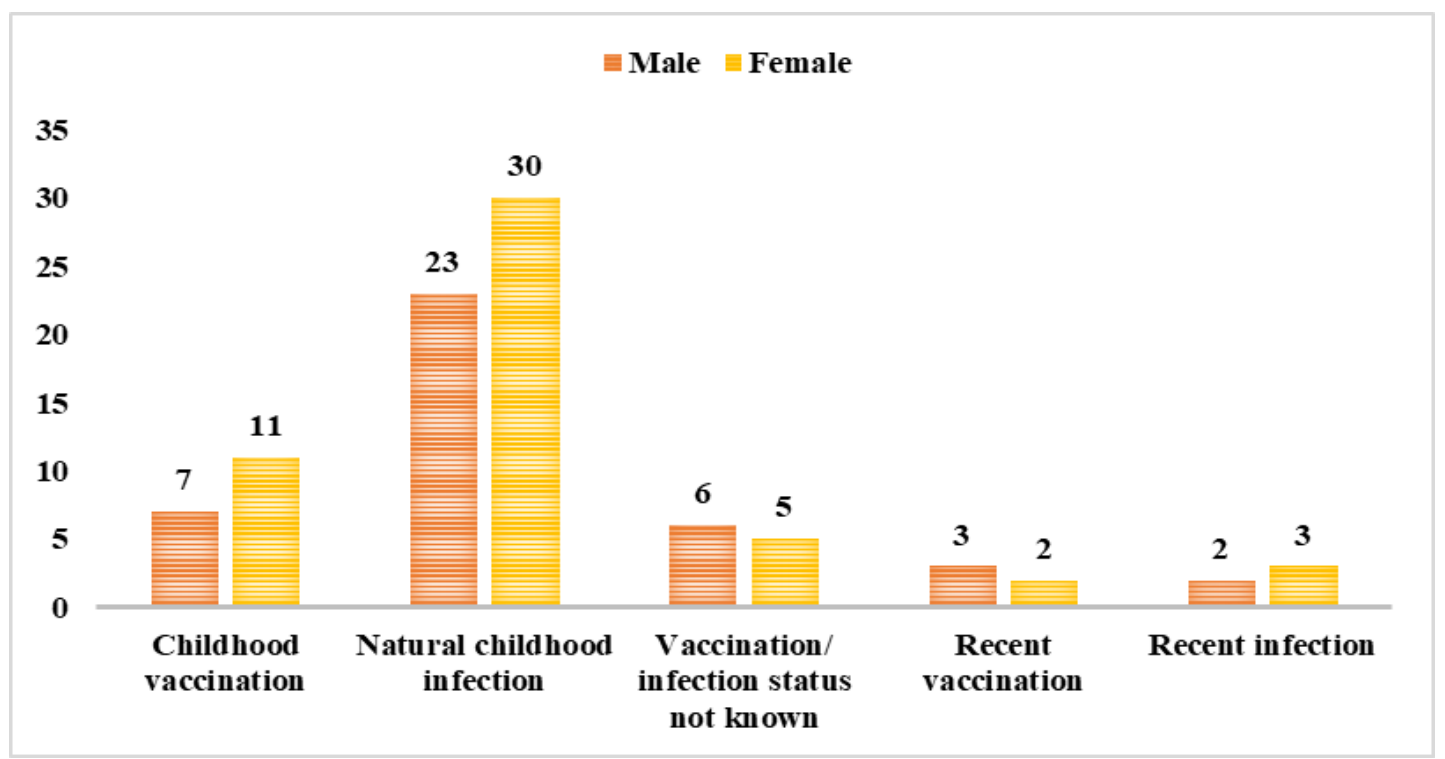

Graph 2: Vaccination of infection status between male and female students.

Table 1: Demographic details

\begin{tabular}{|c|c|}
\hline Year of study & Number of participants \\
\hline $1^{\text {st }}$ clinical year & 24 \\
\hline $2^{\text {nd }}$ clinical year & 36 \\
\hline $3^{\text {rd }}$ clinical year & 22 \\
\hline Interns & 6 \\
\hline Gender & 38 \\
\hline Male & 50 \\
\hline Female & \\
\hline Nationality & 52 \\
\hline Indian & 14 \\
\hline Syrian & 4 \\
\hline Pakistan & 4 \\
\hline Iraq & 3 \\
\hline Egypt & 3 \\
\hline American & 2 \\
\hline Jordan & 2 \\
\hline Canadian & 2 \\
\hline Afghanistan & 1 \\
\hline Bangladesh & 1 \\
\hline Sudan & \\
\hline
\end{tabular}


Based on the history we found that out of 88 students, $19(21.5 \%)$ students received routine vaccination for varicella. 49(55.68\%) students were infected with chickenpox in their childhood. 11(12.5\%) students were unaware of their vaccination and infection status. $4(4.54 \%)$ were infected within five years of the present study, and $5(5.68 \%)$ students got vaccinated recently within 5 years of the present study (Graph 3, 4). What is remarkable is that $13.64 \%(n=12)$ were found to be negative for varicella $\mathrm{IgG}$ antibodies with a mean antibody index 1.76 for varicella antibody titers (Table 2).

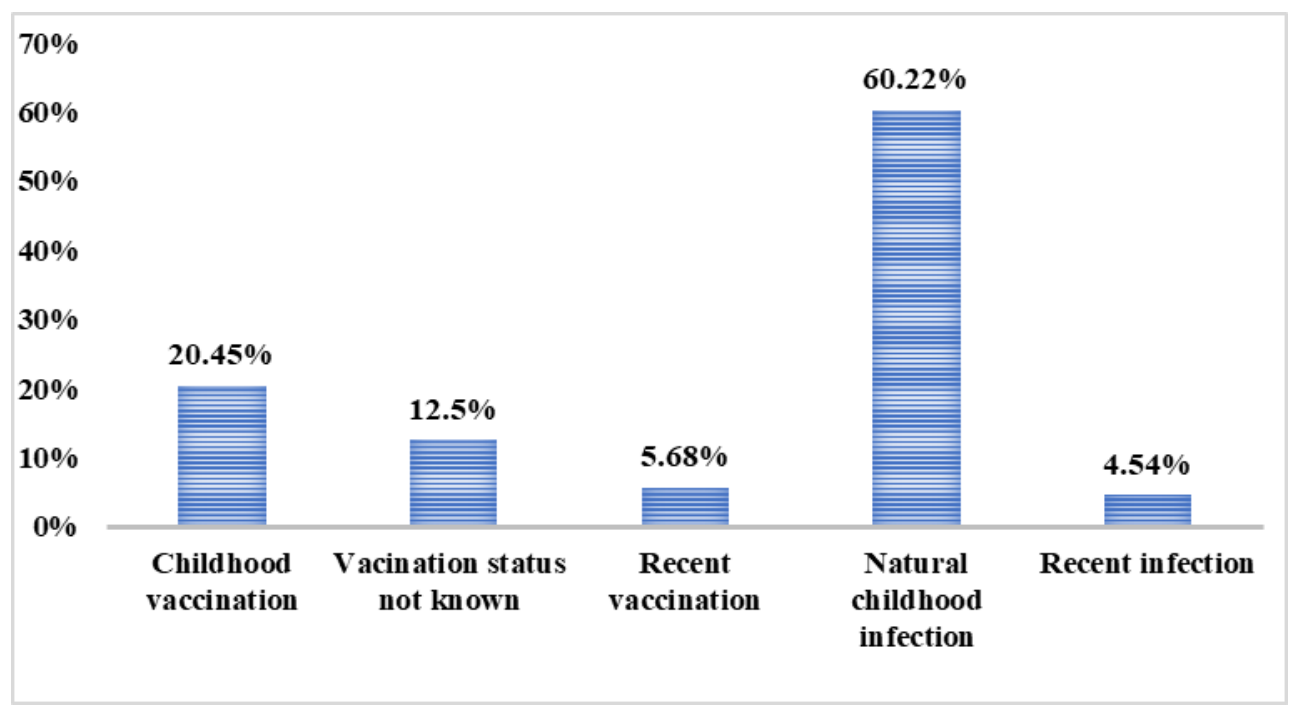

Graph 3: Students with different vaccination and infection status.

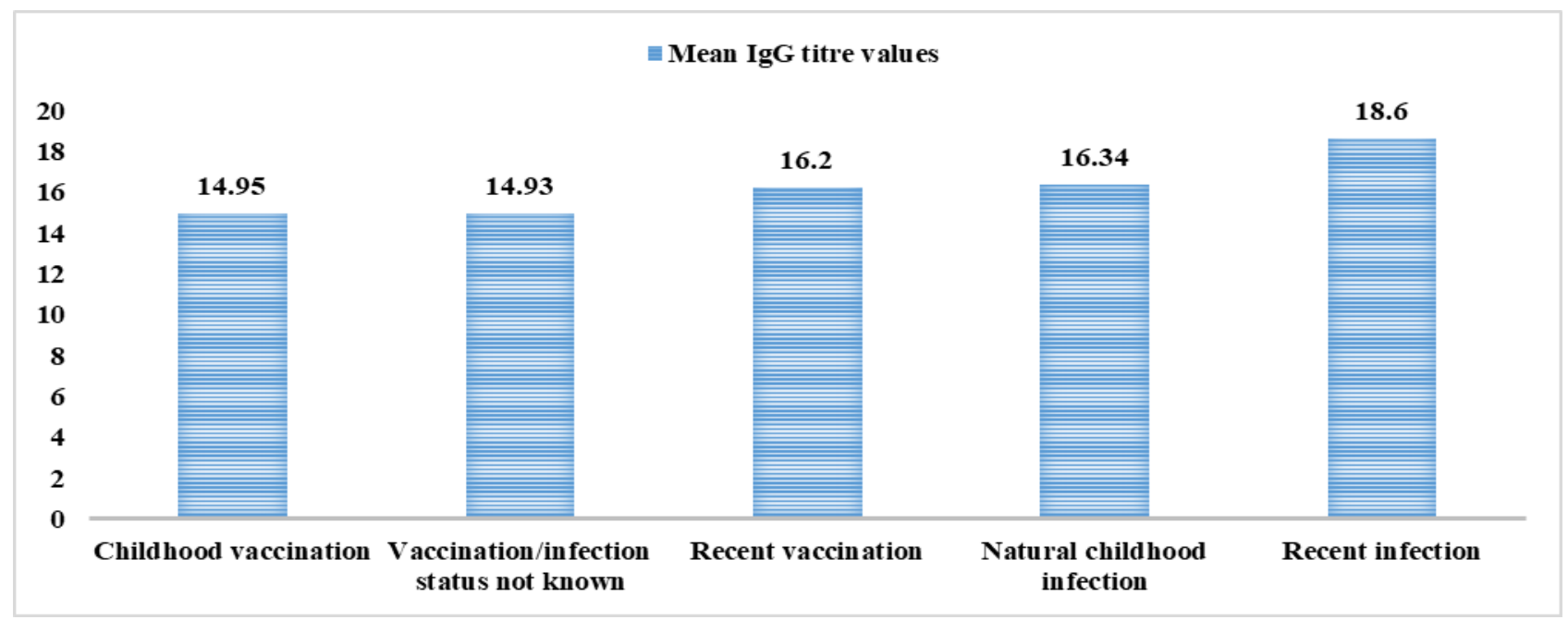

Graph 4: Mean titer values corresponding to vaccination and infection status of students.

Table 2: Seroprevalence among the students

\begin{tabular}{|c|c|c|c|c|c|}
\hline Variable & $\begin{array}{c}\text { Childhood } \\
\text { Vaccination } \\
(n=19) \\
\text { Mean SD }(\%)\end{array}$ & $\begin{array}{c}\text { Vaccination/infection } \\
\text { status not known } \\
(n=11) \\
\text { Mean SD }(\%)\end{array}$ & $\begin{array}{c}\text { Recent* }^{*} \\
\text { Vaccination } \\
(\mathbf{n}=5) \\
\text { Mean SD }(\%)\end{array}$ & $\begin{array}{c}\text { Natural childhood } \\
\text { infection } \\
(n=49) \\
\text { Mean SD }(\%)\end{array}$ & $\begin{array}{c}\begin{array}{c}\text { Recent } * \\
\text { infection } \\
(n=4) \\
\text { Mean SD }(\%)\end{array} \\
\end{array}$ \\
\hline Age & 21.22 & 21.45 & 21.8 & 20 & 21.5 \\
\hline Male & 7 & 6 & 3 & 23 & 2 \\
\hline Female & 11 & 5 & 2 & 30 & 3 \\
\hline Clinical year 1 & 7 & 2 & 0 & 14 & 1 \\
\hline Clinical year 2 & 4 & 7 & 2 & 24 & 0 \\
\hline Clinical year 3 & 7 & 1 & 2 & 11 & 3 \\
\hline Interns & 0 & 1 & 1 & 4 & 0 \\
\hline $\begin{array}{c}\text { Ig } \mathrm{G} \text { antibody } \\
\text { titres }\end{array}$ & $15.04(n=19)$ & $14.8(\mathrm{n}=11)$ & $15.56(n=5)$ & $16.34(\mathrm{n}=49)$ & $18.6(n=4)$ \\
\hline $\begin{array}{l}\text { Antibody } \\
\text { index }<9 \\
\end{array}$ & $2.26(n=3)$ & $1.4(\mathrm{n}=1)$ & -- & $1.62(\mathrm{n}=8)$ & -- \\
\hline $\begin{array}{c}\text { Antibody } \\
\text { index }>11\end{array}$ & $17.44(n=16)$ & $16.17(n=10)$ & $15.56(n=5)$ & $17.88(n=41)$ & $18.6(n=4)$ \\
\hline Total & 19 & 11 & 5 & 49 & 4 \\
\hline
\end{tabular}

$*$ Recent $=$ Within 5 years of the present study 
Those that were infected naturally did not suffer from reinfection, however, 4 students were infected with chickenpox during their clinical years. Out of these 4 students, two of them had received varicella vaccination in their childhood. This is strong evidence that despite getting childhood vaccination, there is no guaranteed immunity against varicella infection. 3 out of 4 recently infected students $(3.4 \%$ ), (who had childhood vaccination )acquired the infection during the course of the study- which is statistically significant.

\section{DISCUSSION}

This study was performed to evaluate the extent of protection to varicella, among the students of a medical university in the UAE to recommend a policy that would ensure their immunocompetence before their hospital training.

VZV infection is considered an occupational hazard for susceptible healthcare workers (HCWs), as it can spread to other susceptible co-workers and patients. Transmission of VZV among HCWs and patients has been reported (1).

In a study done in the year 2019 on Nosocomial transmission of chickenpox and varicella-zoster, the virus seroprevalence rate amongst healthcare workers in a teaching hospital in China showed that Nosocomial transmission of VZV occurred repeatedly in the hospital setting. An alarming 11.6\% of HCWs were seronegative for $\mathrm{VZV}$, which might increase the risk of nosocomial infection and outbreak of infection for other susceptible co-workers and patients. This is especially important in the setting of a teaching hospital where many immunocompromised patients are treated (1).This further reinforces that even the medical students who are a part of teaching hospitals must be checked for protective levels of antibodies against varicella and must be vaccinated if they are not immune to the vaccine-preventable viral infection.

The vaccination against varicella was included in the UAE immunization program in the year 2012, although the vaccine has been available in the UAE before that. And UAE is one of the first countries in the Gulf to have adopted the WHO Expanded Program on Immunization (EPI).

A study done in Los Angeles in 2007 on the Loss of vaccine-induced immunity to varicella over time provided evidence that the protection afforded by one dose of varicella vaccine in children may wane with time. It was found that both the severity and incidence of breakthrough disease increased with the time since vaccination. Children between the ages of 8 and 12 years who had been vaccinated 5 years or more previously were two times more likely to have the moderate-to-severe breakthrough disease. Those who were vaccinated within 5 years have fewer chances of developing the disease irrespective of the age of disease onset or age at which vaccinated. Clinical studies have suggested that the second dose of varicella vaccine could provide increased protection against disease in children by providing protective antibody titers and an improved cellular immune (14).

This brings us to the important point for discussion, which is - immunization protocol for freshmen students in the medical colleges in the UAE'. None of the medical universities in UAE follow such protocol. Since medical students are the ones that are at high risk of acquiring and transmitting infections in hospital settings like other HCWs (Health Care Workers), they need to be protected against these infections, particularly varicella for which there has been found a waning effect in the protective antibody titers with time.

Medical students consistently have sub-optimal immunity to vaccine-preventable diseases, as was highlighted in a recent review of the literature on vaccine coverage among healthcare students (15). Those that have had an infection in childhood and vaccinations before 5 years may still be susceptible due to the waning of antibodies and there could also be a possibility of vaccination failure as well $(16,17)$. All medical sciences students should be tested for Varicella immunity regardless of the history of previous infection $(18,19)$. Quantitative estimation of antibodies will reveal that risk of contacting the desease is much higher than the expected because of the waning effect of childhood vaccinations.

The reason this study was undertaken in 2019 among the medical students of UAE was to estimate the seroprevalence of protective antibody titers against varicella and to recommend to the medical universities of UAE to take up International standard Immunization protocols for freshmen students.

A high proportion of students $60.2 \%$ of students were infected 5 years before the study and $20.45 \%$ of students received vaccination before 2014 . These are individuals at risk of reinfection due to the waning of antibodies (10). $12.5 \%$ of students have never been vaccinated or infected and these carry the highest risk of infection. $4.5 \%$ of students developed injections in their early twenties, that is, after 2014. It is remarkable and must be noted that $13.64 \%$ were found to be negative for varicella antibody titers. These are clinical students who may encounter varicella during clinical rotation and could go on to develop a severe infection after exposure since they have no immunity against varicella. These students must be advised to take live vaccination. Those that were infected or had a natural infection before admission into medical college must undergo seroprotective study and should be advised to take catchup vaccination for protection.

Three students (3.4\%), who had childhood vaccination acquired the infection during the course of our study which is statistically significant. These students are neither immunodeficient nor immunocompromised. 
This shows that even if the students had been vaccinated in childhood, they did not have enough protective antibody titers and thus acquired the infection later in life.

The study spreads light on the fact that despite having childhood vaccinations, medical students are at risk of acquiring vaccine-preventable diseases during their clinical rotations. Catch-up vaccination for susceptible older children and adolescents is of utmost important to prevent the accumulation of susceptibility in these groups, in which the natural disease is more severe (2).

To achieve maximum possible protection for medical students, two policies can be considered: to serologically determine immunity status and selectively vaccinate susceptible individuals, or, to blindly vaccinate all students without prior serology.

The cost minimization results indicate that the first approach is most suitable for varicella which is to determine immunity status serologically (20). For students who prefer not to receive any unnecessary vaccine, it is always possible to identify need for vaccination by perfoeming serological tests. Vaccination can be undertaken in the case of negative antibody titers.

Other health services and education providers must reflect on their current immunization policies and take action to protect the health of their students and the wider community. This will ensure the students' immune status and decrease the risk of contracting or transmitting infection (11).

\section{CONCLUSION}

This study highlighted the absolute need to address the vaccination rates of medical students, a population that should be extremely motivated to ensure that their immunity to common vaccine-preventable diseases is optimum not just their safety but also for the safety of the patients they will be contacting who can potentially be immunocompromised. This study demonstrates that there is a high proportion of medical students that are at risk of acquiring varicella due to the nature of their occupation .Furthermore, since these students are in early adulthood, they can have more serious complications after being infected. The students at the highest risk are those that have not been vaccinated or have never had natural infection. The only students that are at least risk are those that have been vaccinated recently, during or just before their clinical years.

The infection and complication among clinically exposed medical students can be minimized and even eliminated if Universities in the Asian countries adapt to, protocols followed by wetern Universities that is mandatory requirement of updated immunization record that ensures the protective titers for medical school admission .
We recommend all medical universities of UAE and other Asian countries must have a mandatory check on the immunization status before admission into the first year at par with western international admission protocols. This will be beneficial to the healthcare system, have a protective impact, and can play a role in legal implication.

\section{ACKNOWLEDGMENT}

Authors are grateful to Dr. Gurumadhava Rao, President RAKMHSU for allotting funds, and encouraging in conducting the study.

\section{CONFLICTS OF INTEREST}

The authors declare no conflict of interest.

\section{REFERENCES}

1. Yang, J., Liu, J., Xing, F., Ye, H., Dai, G., Liu, M., et al., Nosocomial transmission of chickenpox and varicella zoster virus seroprevalence rate amongst healthcare workers in a teaching hospital in China. BMC Infectious Diseases. 2019; 19(1).

2. Marin, M., Nguyen, H., Keen, J., Jumaan, A., Mellen, P., Hayes, E., et al., Importance of catch-up vaccination: experience from a varicella outbreak, Maine, 2002-2003. Pediatrics. 2005; 115(4): 900-905.

3. Gustafson, T. L., Lavely, G. B., Brawner, E. R., Jr., Hutcheson, R. H., Jr., Wright, P. F., Schaffner, W. An outbreak of airborne nosocomial varicella. Pediatrics. 1982; 70: $550-556$.

4. Dogger-Goren, S., Daba, K., Hurley, P., Yabuuchi, H., Takahashi, M., Ogra, P. Antibody response to varicella-zoster virus after natural or vaccine-induced infection. The Journal of Infectious Diseases. 1982; 146(2): 260-265.

5. Watson, B. Humoral and cell-mediated immune responses in healthy children after one or two doses of varicella vaccine. The Pediatric Infectious Disease Journal. 1995; 14(6): 556.

6. Kroger, A. T., Atkinson, W. L., Marcuse, E. K., Pickering, L. K. General recommendations on immunization: recommendations from the advisory committee on immunization practices. Pediatrics. 2007; 119(5): 1008-1008.

7. Sheek-Hussein, M., Hashmey, R., Alsuwaidi, A., Al Maskari, F., Amiri, L., Souid, A. Seroprevalence of measles, mumps, rubella, varicella-zoster and hepatitis $\mathrm{A}-\mathrm{C}$ in Emirati medical students. BMC Public Health. 2012; 12(1).

8. Al-Turab, M., Chehadeh, W. Varicella infection in the Middle East: Prevalence, complications, and vaccination. Journal of Research in Medical Sciences. 2018; 23(1): 19.

9. Arunkumar, G., Vandana, K., Sathiakumar, N. Prevalence of measles, mumps, rubella, and varicella susceptibility among health science students in a University in India. American Journal of Industrial Medicine. 2012; 56(1): 58-64.

10. Glass, K., Grenfell, B. Antibody dynamics in childhood diseases: waning and boosting of immunity and the impact of vaccination. Journal of Theoretical Biology. 2003; 221(1): 121-131.

11. Fergus, E., Speare, R., Heal, C. Immunisation rates of medical students at a tropical queensland university. Tropical Medicine and Infectious Disease. 2018; 3(2): 52.

12. Loulergue, P., Launay, O. Vaccinations among medical and nursing students: Coverage and opportunities. Vaccine 2014: 32: 4855-4859.

13. World Health Organization. Summary of WHO position papers-immunization of health care workers; World Health Organization: Geneva, Switzerland, 2017.

14. Chaves, S., Gargiullo, P., Zhang, J., Civen, R., Guris, D., Mascola, L., et al., Loss of vaccineinduced immunity to Varicella over time. New England Journal of Medicine.2007; 356(11): 1121-1129. 
15. Loulergue, P., Launay, O. Vaccinations among medical and nursing students: Coverage and opportunities. Vaccine. 2014; 32: 4855-4859. doi: 10.1016/j.vaccine.2014.01.014

16. Verstraeten, T., Jumaan, A., Mullooly, J., Seward, J., Izurieta, H., DeStefano, F., et al., A retrospective cohort study of the association of varicella vaccine failure with asthma, steroid use, age at vaccination, and measles-mumps-rubella vaccination. Pediatrics. 2003; 112(2): e98-e103.

17. Heininger, U., Bachtiar, N., Bahri, P., Dana, A., Dodoo, A., Gidudu, J., et al., The concept of vaccination failure. Vaccine. 2012; 30(7): 1265-1268.

18. Almuneef, M., Memish, Z. A., Abbas, M. F., Balkhy, H. H. Screening healthcare workers for Varicella zoster virus: Can we trust the history? Infect Control Hosp Epidemiol. 2004; 25(7): 595-598. http://dx.doi.org/10.1086/502445.

19. Baer, G., Bonhoeffer, J., Schaad, U. B., Heininger, U. Seroprevalence and immunization history of selected vaccine preventable diseases in medical students. Vaccine. 2005; 23(14): 2016-20.

20. Kimmel, S. R. Vaccine adverse events: separating myth from reality. Am Fam Physician. 2002; 66: 2113-2120. 